\title{
Contributions of Training Programs Supported by VR Techniques to the Prevention of STF Accidents
}

\author{
Anika Weber ${ }^{123}$, Peter Nickel ${ }^{3}$, Ulrich Hartmann ${ }^{1}$, Daniel Friemert ${ }^{1}$ and Kiros \\ Karamanidis $^{2}$ \\ ${ }^{1}$ Koblenz University of Applied Sciences, Department of Mathematics and Technology, Rema- \\ gen, Germany \\ ${ }^{2}$ London Southbank University, School of Applied Sciences, Sport and Exercise, \\ Science Research Center, London, UK \\ ${ }^{3}$ Institute for Occupational Safety and Health of the German Social Accident Insurance (IFA), \\ Department of Accident Prevention: Digitalisation - Technologies, Sankt Augustin, Germany \\ weber4ahs-koblenz. de
}

\begin{abstract}
Occupational safety and health (OSH) is active at all levels of the hierarchy of controls to prevent accidents associated with slips, trips and falls (STF). Training programs related to STF prevention are increasingly supported by virtual reality (VR) techniques. A review revealed a wide range of applications in practical and scientific areas. Trainings for operational practice vary regarding objectives, target groups, application contexts, media, and effectiveness, if available. Trainings in scientific studies are well designed for specific purposes at hand, but not suitable for direct application in operational practice. Research is required to bridge the gap. An investigation on gait stability and control in a VRbased obstacle avoidance training scenario has been conducted to contribute to developments in STF prevention. Initial results indicated a high level of presence and no evidence for detrimental effects on body and gait stability through application of VR techniques. This provides a sound basis for analysis of other data still required and for guiding similar and subsequent studies along knowledge gained by training programs available.
\end{abstract}

Keywords: Behavioral training $\bullet$ Gait perturbation $\bullet$ Virtual reality $\bullet$ Slip, trip, and fall hazards $\bullet$ Occupational Safety and Health

\section{Introduction}

The prevention of accidents involving slips, trips and falls (STF), accounting for about $20 \%$ of all accidents across national studies, is at high priority in occupational safety and health (OSH) [1]. The selection of prevention measures should preferably follow the internationally established hierarchy of controls (STOP model) [2,3]. By means of substitution measures (e.g. using alternative walking routes without obstacles) or technical measures (e.g. designing work areas without steps, laying anti-slip floors), STF hazards are eliminated in principle and for many employees at the same time. In addition, organizational (e.g. tidying up work areas, walking on official paths) and personal 
measures (e.g. wearing work shoes as personal protective equipment, attending behavioral training programs to reduce the consequences of accidents) can also contribute to protection of employees and improvements in OSH.

Behavioral prevention measures such as training in STF prevention have been discovered for virtual reality (VR) applications and are increasingly popular [4]. It seems rather easy to draw employees' attention to, to sensitize employees to STF hazards, to teach them how to behave safely and healthy and to inform them about strategies and skills for STF prevention or reducing accident severity. In addition, it is assumed that implementation and use of VR in training applications will reduce the amount of physical training equipment required, increases flexibility in terms of training location and time, offers high fidelity training scenarios with hazardous situations at stake, and allows for new interventions for implementing training content. Some of the studies on development and implementation of VR in training environments demonstrate, however, that it might not always be that easy $[5,6]$.

However, research on the relationship between gait stability as well as coordination and STF give rise to the assumption that motor trainings have the potential to improve stability and coordination even in STF situations (see Sect. 2.3 and e.g. [7]). In addition, studies on walking on treadmills show significantly more natural human behavior when immersed in virtual scenarios as compared to without virtual environments (VE) [8]. Some adult education is rather open-minded towards new methods and media. Development of concepts such as assisted learning [9] offer a framework for integrating new methods or media into training procedures so that technical support for educational methods match didactics to improve learning.

Several training programs have already been developed in the context of STF prevention and the number of trainings that integrate VR technique increases. A review of training programs in the context of STF hazards that integrated VR techniques resulted in a collection with differences in objectives, content, structure and information on the effectiveness in the area of STF prevention. Results will be discussed and a strategy for setting up a new training program will be developed based on advantages and limitations of trainings available.

\section{Review of Training Programs}

\subsection{Procedure for Research and Classification}

A review of existing training programs was conducted to collect information about their objectives, target groups, application context, media representation, interventions and statements on effectiveness. The review focused on training programs that referred to potential STF hazards and involvement of VR techniques by means of an internet search with different search engines and combinations of terms (e.g. "training", "tripping", "virtual", "STF") in German and English language.

Two fundamentally different groups of results were identified. On the one hand, several training applications supported by VR techniques and offered for operational practice were found that addressed organizations on OSH, VR service providers, training providers and consulting firms were found. On the other hand, there were references 
to scientifically oriented studies that analyzed behavioral parameters for body stability and coordination in virtual scenarios or examined effects of variations in training on human gait parameters.

The findings provided a broad range of information with variations across training programs. However, based on information given, it was not always clear whether the training program was focused on training, on application with VE, on OSH or something different. Not all training programs presented information about a rationale or intended use. With the aim to structure the contents, an attempt was made to present information on objectives, target user groups, application context, media and VR techniques required, possible interventions and statements on effectiveness.

\subsection{Trainings Supported by VR, Related to STF Prevention and Operational Practice}

Work Safe, Texas - Safety in a Box for Construction and Production Safety [10]. The Work Safe training program allows employees to virtually experience deadly and serious accident hazards on construction sites: Falls from height; Struck by an Object; Electrocution; and Caught In or Between. The training program for building safety is similar to production safety. Users virtually experience three safety relevant scenarios that are common in a production environment: Lockout/Tagout When Working with Machinery; Slips, Trips and Falls; and Forklift Awareness. The program requires employees to watch $360^{\circ}$ videos on construction and production safety in a Cardboard VR. With several scenarios available, each concludes with a multiple-choice quiz. Employees get feedback, they are informed about potential consequences and are presented appropriate solutions or behaviors.

Plonsker Media / BG Bau - Fall Protection on the Construction Site [11, 12]. An occupational safety training, developed for the German Social Accident Insurance Institution for the building trade, intends to train and instruct employees on a range of topics relevant in OSH in the building trade. In this training program, VR serves as a medium for integrating more naturalistic environmental conditions in training scenarios. The application demonstrates a range of personal protective equipment (PPE) (e.g. helmet, belt) for fall protection. The selection of suitable measures for fall protection (e.g. closing flaps of scaffoldings, attaching cross braces to scaffoldings, putting on PPE, attaching spring safety hooks to a safety rope) is rewarded with tokens to be collected by the user. The user wears a commercially available VR headset, can move freely in space and interact with objects in the virtual environment using controllers.

Safety-and-Health-at-Work - Working Height Safety [13]. The training program intends to train employees on safely working at height. For virtual work on an inspection hood of an air conditioning system on a high roof, equipment (e.g. personal fall distance limiter), colleagues (e.g. full-body harness) and the employee itself (e.g. safety rope attached) are virtually checked and asked to climb up a ladder to a sloping roof. A scenario is available in which the force applied by the worker to the hood is too great 
and the user falls backwards over the edge of the roof being restrained by the personal fall distance limiter. The simulation in the VE used in the training program allows different views: first person, observer from near range and from ground level. Navigation in virtual scenarios is carried out with the aid of a commercially available VR headset and two controllers.

American Society of Safety Professionals - Fall Protection Training Tool [14]. The training program intends to train good operational practice through an application in a fall prevention training that is supported by VR techniques. Employees use an app to train in realistic work scenarios without endangering themselves. In the VR application, hazards should be identified and fall protection systems should be selected. A roof scenario is presented with several fall hazards, such as tripping hazards due to devices close to the edge of the roof and open roof windows. Employees are required to assemble a safety system for colleagues. Anchorage points, harnesses and ropes should be selected suitable to serve as protective safety system in the situation. The fall scenario varies with the choice of the assembled safety system.

Energy.Gov - Slips Trips and Falls [15]. This training program is designed to teach workers how to move in work scenarios with various hazards before facing hazardous situations in facilities in reality. Employees walk through a virtual scenario and avoid safety risks (e.g. tripping hazards, piping above floor level, low hanging infrastructure). They identify dangerous objects in their way and remove them, so they have a walking and working area without hazards. While walking in the scenario, trackers are used to record body movements of the employees. Employees are given points for eliminating hazards or hazardous situations. This adds an entertaining video game element to the training program.

BGHW Mobile "More Safety Through Personal Experience" - Warehouse Simulator [16, 17]. A showtruck of the German Social Accident Insurance Institution for the trade and logistics industry includes several areas for OSH related activities. At one activity station, employees walk through various virtual rooms of a logistics center in search of tripping and slipping hazards, wearing a commercially available VR headset. The aim of this simulation application supported by VR techniques is to find as many hazards as possible, to select suitable measures to prevent them and to receive feedback about chosen activities. Among others, the application should be used to get into conversations with employees and to make them realize that each employee can always do something for their own safety and the safety of colleagues in the company (e.g. from picking up objects lying around to informing the supervisor about hazards).

VR Health and Safety Training - Slips, Trips and Falls [18]. This training program advertises that the training of critical safety standards should leave a lasting impression. The VR-based training program intends to improve training effectiveness and training transfer. In a playful way, the employees are challenged to identify typical hazards in a 
simulated workplace environment. Employees will get feedback about their activities including information about what should have been done. Health and safety training topics include topics like fire alarm or slips, trips and falls. A video also shows the use of PPE (e.g. helmet, safety goggles and gloves).

IBEW - Slips, Trips \& Falls [19]. This training program among others, concerns with slip, trip and fall hazards and allows prospective trainees to explore a construction site virtually via a $360^{\circ}$ application with a smartphone or a commercially available VR headset. Five one-hour modules show different typical dangers (e.g. module slips, trips and falls). Danger points in the virtual environment are marked with symbols. When the trainee selects a danger point by clicks, the application presents detailed information about relevant hazards. The program was tested in a training center with 20 trainees. Young trainees accepted the VR learning technology better compared to teaching in traditional training and demonstration style.

EdgVR@ Health \& Safety - Slips, Trips and Falls [20]. The training program includes $360^{\circ}$ photos, videos and an interactive platform and among others, presents OSH topics such as slips, trips and falls. This training program is designed to be used with commercially available VR headsets that allows up to 35 users to act independently of each other at the same time. The system intends to detect participants' potentially relevant needs for further training in OSH or required professional knowledge on safety and health issues for a range of task.

\subsection{Trainings Supported by VR, Related to STF Prevention and Scientific Research}

In addition to practical or commercial offers for training programs with VR support, scientific studies also reported in the context of STF prevention, are often associated with biomechanical motor training programs. Almost all of the training programs have an interest in slipping behavior, i.e. with virtual slipping imposed by tilting the image of the VE presented in VR glasses [21-29].

Results of the studies showed that participants walk more carefully in VE compared to walking outside VE; as indicated by reduced gait speed, shorter strides, longer double support, and higher step width [25, 24, 28, 23]. Therefore, it could be assumed that just walking while present in a VE and without virtual perturbations, challenges gait stability and coordination. Virtual perturbation (e.g. by tilting of the image) added to walking in VE, sometimes led to improvements in gait and coordination parameters [25, 28]. In addition, VR support in trainings where participants went shopping in VE under different conditions (e.g. icy, virtual tilt of the environment, stepping over virtual obstacles, multitasking) led to lower fear of falling [21].

In training programs, it could be demonstrated that participants showed reactive and proactive adjustments to tilting VE. Moreover, when transferred to real slipping situations, participants improved interception strategies even without training in real situations [22, 23]. If this training program would indicate long-term effects and were to be 
transferred into real life scenarios with participants of the general public (e.g. rehabilitation or prevention centers), it may have the potential to reduce the risk of high accident severity in some fall situations.

Since medio-lateral oscillations of the visual field cause greater perturbations of gait than anterior-posterior oscillations [26], alternative procedures have been investigated to simulate slipping experiences in VE. One study showed that perturbations by tilting in the roll plane of a treadmill in the virtual environment with or without physically moving the roll plane of the real treadmill (standing on a 6 DOF motion base) resulted in no significant differences in gait stability and coordination [24]. Although researchers concluded that perturbations initiated by VR systems could generate a cost-effective approach for gait rehabilitation in patients, it seems to be long way for development of an effective and valid training program in this setting. Another study concluded that VE could produce results in fall interventions comparable to training programs with a moveable platform [27]. Besides similar effects in reductions of fall frequencies for both environments, differences occurred for body motions. While virtual slips initiated by tilts in the pitch plane of the VE led to an initial response in the upper body and a secondary response in the lower extremities, slips initiated by the moveable platform led first to a response in the lower extremity and then to a response using hip and trunk. Consequently and although some final effects seemed to be comparable using the moveable platform and the VE [27], differences in motor behavior rather suggested requirements for different compensatory strategies and therefore different behavioral control for fall prevention.

Recently, VE have also been used to train obstacle negotiation [30]. Therefore, participants walked through a virtual corridor and occasionally crossed virtual obstacles. After crossing obstacles, they got feedback about the distance between foot and obstacle by pleasant and unpleasant sounds, depending on close range crossing and out of range crossing of the obstacle. Thus, participant learning to cross the obstacle as close as possible was reinforced. As a result, they found that participants successfully reduced foot clearance as instructed during practice trials, transferred the reduced foot clearance to over-ground obstacle negotiation, and retained the reduced foot clearance after $24 \mathrm{~h}$ [30].

\subsection{Evaluation of Both Groups of Training Programs}

In principle, objectives of the above-mentioned training programs for the research sector (see Sect. 2.3) are relatively easy to understand compared to those for operational practice (see Sect. 2.2). However, illustrations and explanations for practical training programs more closely connect to practical situations at work and more obviously are interested in prevention of STF hazards and accidents.

Each of the above-mentioned training programs related to workplace practice (see Sect. 2.2) aims to contribute to the prevention of STF accidents or at least to reducing their severity. However, due to a lack of information, it is not possible to determine how and to what extend this is feasible and whether the content of the training program contributes to the objective. Potential contributions or results for employees are, among 
others, informing about hazards, identifying danger points, showing possible courses of action and experiencing dangerous situations without putting oneself in danger.

It is assumed, however, that (1) contributions actually take place, i.e. relevant training participants recognize, understand and take on information given and actions can become effective in the population and therefore, (2) training participants back in real life situations, actively influence STF situations so that near misses and accidents are prevented. An evaluation for both requirements is necessary, before a training program could be assumed to be relevant and significant in the field of STF prevention. Two statements from descriptions of the training program in Section 2.2 provide hints at potential evaluation results. One the one hand, it is mentioned, that interest in VR techniques or related media was more interesting compared to classical teaching. Unfortunately, this does not necessarily result in improved effectiveness of training programs. On the other hand, it was pointed out, that studies have shown increases in retention of training content with increasing fidelity of the training environment. Unfortunately, due to lack of reference to an evaluation study and direct referencing to the training program itself, this statement cannot be generalized for all training programs. Nevertheless, practical training programs may have in common that they intend to train participants in identification and elimination of STF hazards.

None of the training programs mentioned in relation to research (see Sect. 2.3) pursues the goal of conclusively developing an effective measure for preventing STF accidents. Rather, the aim is to investigate individual or several behavioral-preventive cause-effect relationships on the path between gait stability and coordination parameters and a potential contribution to reductions in the severity or prevention of STF accidents. Aim of investigations therefore should be the effectiveness of training programs with and without VR support for intended variations in relevant gait parameters including information about the generalizability of results. In addition, transfer of training effects to real life situations should become available and be maintained in the long term. Finally, it should be possible to show that in situations with STF hazards, accidents can be prevented, or their severity reduced.

From the overview of research activities as documented in Section 2.3, however, it is obvious that evidence suitable for training programs in STF prevention is rather limited. The same is true for information available about cause-effect relationships between gait stability and coordination and STF. At the same time, it is also evident that development and implementation of effective training programs for STF prevention appear to be much more complex and seem to require more than just VR techniques than offers available for operational practice would suggest.

\subsection{Discussion}

An increasing number of training programs is available with close links to a rather broad topic like rehabilitation and prevention of STF and OSH. Even if training programs supported by VR techniques range at lower level in the hierarchy of controls for improving working conditions according to OSH [2], they have the potential to support prevention and actively involve employees in activities for prevention of STF accidents. 
However, research activities should continue to focus on cause-effect relationships between gait stability and coordination parameters and potential contributions to reductions in the severity or prevention of STF accidents. Operational practice should continue to involve in development and implementation of training programs and support the transfer of evidence available to the shop floor level. While high fidelity usually does not result in impairing effects for training programs supported by VR techniques, it is a rather unreliable predictor for immersion and the feeling of presence in VE [5]. In addition, simply developing a VE does not imply that it is good enough to achieve training gains [6].

Unfortunately, the review of already existing training programs was not systematic, so that not all existing training programs could be included. Nevertheless, the programs identified indicated significant differences in objectives, content, structure and information on effectiveness in this area of accident prevention. Training programs related to operational practice have not yet been able to demonstrate and inform about their contribution to prevention of STF accidents. They are nevertheless valuable because they attempt to depict working conditions in relevant scenarios at work and provide visual support for virtual identification and elimination of STF hazards.

Research-based training programs have not yet been able to develop solutions for operational practice although some investigations suggest or believe that the results may significantly contribute to training relevant in practice. Based on training programs presented, it is clear that there are currently more open questions than satisfactory answers. However, results of the review are encouraging and initial achievements increase confidence that ongoing research activities may contribute to prevention of STF accidents.

The review already provides some indications for strategies for further research. A good starting point is provided by a preliminary study that aims to examine learning effects of a virtual obstacle avoidance training on gait stability in young adults (see Sect. 3). Further studies should follow, with investigations into transfer of results to trip hazards in reality and into short as well as long-term effects for gait stability and coordination trainings supported by VR techniques relevant for different age groups and scenarios related to operational practice.

\section{Study on Obstacle Avoidance supported by Virtual Reality Techniques}

The prospective study is part of a research agenda with the aim to investigate training procedures with long-lasting effects on gait stability and coordination. The training should be suitable to a broad range of working population and should apply VR techniques to support training procedures. The review of already existing VR training programs for STF prevention shows that VR techniques could be helpful to support training programs. Results also provide some indications for strategies for further research. An initial study will investigate whether gait stability of young adults can be improved 
by training of obstacle avoidance in VE. Furthermore, it was investigated whether potential adaptation to repeated disturbances of one leg can promote better performance of crossing obstacles with the untrained leg.

Future studies should follow, with investigations into transfer of results to trip hazards in reality and into short as well as long-term effects for gait stability and coordination trainings supported by VR techniques relevant for different age groups and scenarios related to operational practice. The whole research project tries to investigate cause-effect relationship between gait stability and coordination and STF.

\subsection{Methods}

Participants. Twenty-four young and healthy participants $(M=22.3$ years, $S D=2.4)$ provided informed consent to take part in empirical investigations. All participants reported high (corrected) visual acuity and no impairments with regard to walking and body movements.

Task environment. The investigations were conducted in the Laboratory for Biomechanical Studies, RAC facilities of Koblenz University of Applied Sciences. A treadmill (Pluto, $\mathrm{h} / \mathrm{p} / \mathrm{cosmos}$, Germany) with mechanical fall arrester was placed in the center of the laboratory. While three cameras for motion capturing (Opus, $120 \mathrm{~Hz}$; Qualisys $\mathrm{AB}$, Sweden) were set up at ground level close to the treadmill, five cameras were installed on rails attached to all sides of the ceiling. In addition, of the four lighthouses for head-tracking of a VR headset (Vive Pro, HTC, Taiwan) three were mounted to the rails and one was placed on a tripod close to the treadmill.

On the treadmill, participants walked in a virtual environment along a corridor and were occasionally required to cross obstacles presented in the scenario. For orientation in the scenario, a 3D model of the treadmill was integrated and the participants' body was virtually visualized by an avatar (Fig. 1). Unity (Unity Technologies ApS, USA) served as a software system for VR programming and was used to develop the virtual environment. This system and the motion capturing system were synchronized and used the same coordinate system. The motion capturing system tracked dynamic body, leg and foot postures from markers attached to the participants to feed analyses of parameters for body and gait stability. In addition, participants' foot positions in gait cycle were analyzed to deliver a signal to the VR system that triggered stimulus presentations in VR (virtual obstacle). 


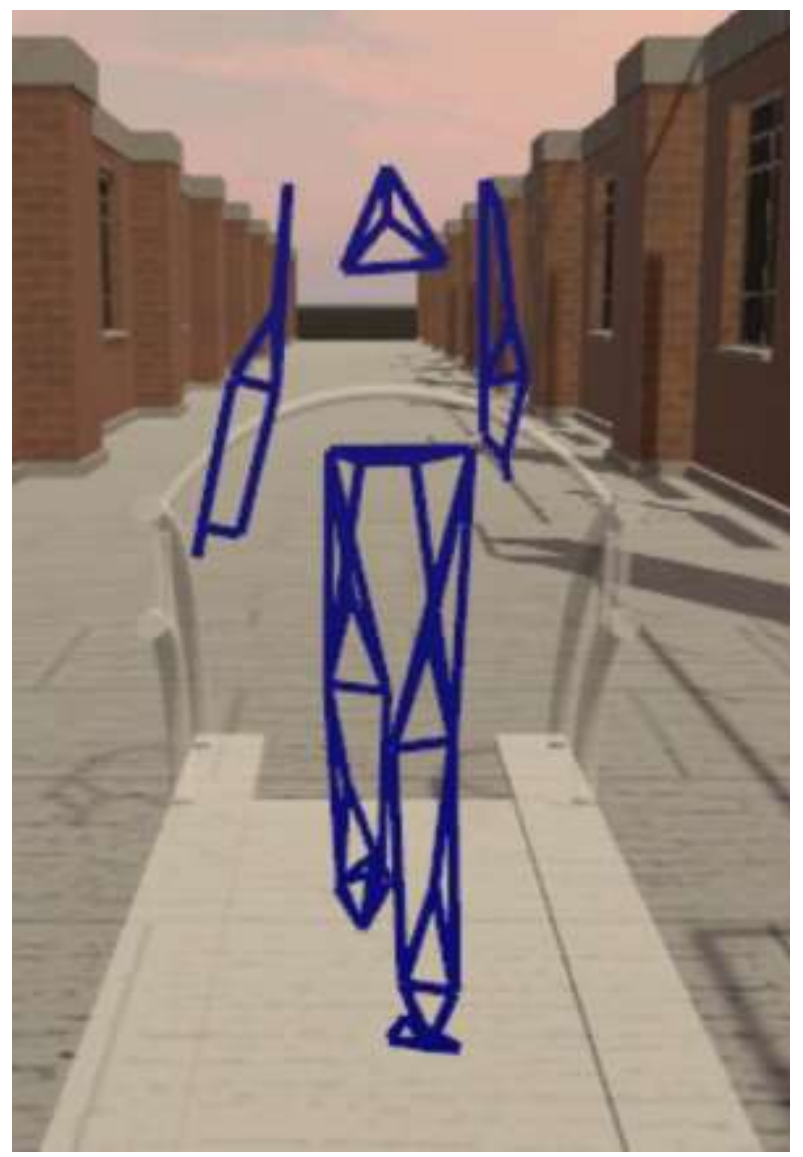

Fig. 1. Experimenters view of VE from behind at participant (blue avatar) on treadmill (overlay included) while walking along a suburban area. Participants' point of view is the avatars' view.

Experimental Procedure and Study Design. The experiments lasted about $120 \mathrm{~min}$ each, subdivided into consecutive sections for briefing and preparation including initial questionnaires, one session either in the control group or in the intervention group, and debriefing including another assessment with questionnaires. At the beginning, participants were asked to provide informed consent and were informed about their rights as participants. They filled in questionnaires on demographics and baseline performance. Before start of session, both groups were able to familiarize with the equipment by walking on the treadmill without and with wearing a VR headset.

Next, the intervention group started their training session while walking on the treadmill and crossing 51 virtual obstacles (width 500, height 100, depth $100 \mathrm{~mm}$ ) with one leg only. Obstacles appeared unexpectedly in the virtual scenario in front of the participants. The control group walked on the treadmill for $10 \mathrm{~min}$ without obstacles and was then required to cross 16 virtual obstacles. Total time of both groups on the treadmill 
with VR was the same. The ability to successfully cross obstacles required integration of visual information about the virtual environment and self-assessments of participants own body posture (see Fig. 1).

In the briefing phase, all participants completed questionnaires on simulator sickness (SSQ) [31, 32] and immersion tendency (ITQ) [33]. During the sessions, measures of participants body and gait stability were acquired based on 48 markers attached to foot, leg, body, head and VR headset that were tracked by the tracking system. In the debriefing phase, all participants completed questionnaires, such as SSQ, Presence Questionnaire (PQ) $[33,34]$ and on perceived mental workload by using the task load index (NASA-TLX) [35]. All questionnaires were presented on a Laboratory computer using LimeSurvey (LimeSurvey GmbH, Germany) as an online survey tool.

Since analysis of biomechanical measures on body and gait parameter is not yet available, analysis refers to questionnaire data only. Therefore, data from sessions do not require repeated measures analysis. With the number of participants balanced for the control $(N=12)$ and intervention $(N=12)$ group, a non-randomised controlled trial design is applied (t-Test; IBM® SPSS ${ }^{\circledR}$ Statistics 23 , USA) to questionnaire results. For all statistical tests, the significance level was set at $p<0.05$.

\subsection{Results}

Simulator Sickness. The questionnaire on simulator sickness was rated before and after the session of both control and intervention group. The analysis of difference measures resulted in minimal symptoms for the control group $(M=8.74 ; S D=12.82)$ and negligible symptoms for the intervention group $(M=2.19 ; S D=3.38$ ) (Fig. 2) according to sickness score classifications [36]. Statistical analyses revealed no differences between groups.

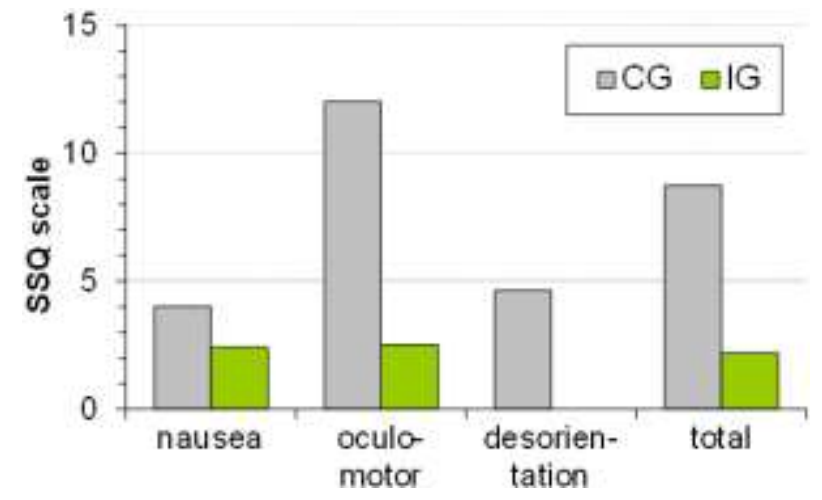

Fig. 2. Results for SSQ sub-scales and total scales for control (CG) and intervention group (IG).

Sense of Presence. It is recommended to investigate the tendency of participants to experience presence before an assessment of presence should take place [32]. Analyses 
of the ITQ revealed no differences for the control and the intervention group, neither for the total level of immersion tendency $(M=85.17$; $S D=10.33)$, nor for sub-scales such as tendencies to become involved in activities $(M=29.96 ; S D=7.77)$, to maintain focus on current activities $(M=38.17 ; S D=3.77)$ and to play computer games $(M=$ $8.17 ; S D=3.36)$. Results of the present study are very similar to average values across other studies [33].

The sense of presence (PQ) in the control and in the intervention group yielded no differences between groups, however, an internationally comparable and high level for the total value $(M=125.88 ; S D=22.69)$ as well as for the sub-scales involvement $(M$ $=56.04 ; S D=12.19)$, sensory fidelity $(M=20.63 ; S D=8.33)$, adaptation/immersion $(M=43.17 ; S D=6.13)$, and interface quality $(M=6.04 ; S D=2.18)$.

Perceived Mental Workload. The sequence of task processing did not reveal any significant influence on the results of this evaluation study. Mental workload during the sessions was rated under-average in the control group $(M=24.64 ; S D=8.08)$ and in the intervention group $(M=29.58 ; S D=9.96)$. Statistical analysis yielded no significant differences between groups.

\subsection{Discussion}

The study presented intends to investigate learning effects of virtual obstacle avoidance training on gait stability in young adults. Unfortunately, analysis of biomechanical data was not undertaken yet. Procedures for data analysis have already been presented [37] and results for the present study are in preparation [38]. However, positive results could be obtained from the pre and post examinations with questionnaires on relevant dimensions in training studies in virtual environments.

Across all these measures, results suggested no detrimental effects by participating in the study and demonstrated that no differences could be identified between control and intervention group according to statistical analysis of simulator sickness, immersion tendency, feeling of presence and mental workload. In addition, perceived mental workload was rated rather low in general and the feeling of presence was at high level; similar to those reported in the literature [33].

This provides a sound basis for analyses of biomechanical data and for guiding more elaborated training studies along the lessons learned and experiences gained in initial studies. Occupational health and safety should therefore proceed in follow its long tradition in simulation research to use its specific advantages.

\section{Conclusions}

Regarding the number of accidents related to STF hazards, application as well as development of suitable measures are very welcome. Although the hierarchy of controls is in favour of the strategy to prefer measures at upper levels, all hierarchy levels are required to contribute effectively. Regarding training programs available more information and exhaustive evidence would be required to be able to choose a program that 
covers demands in operational practice and demonstrates compliance with OSH requirements. This calls for evaluation studies on information quality and on potential contributions for the prevention of STF accidents at shop floor level. Trainings supported by VR techniques and related to STF prevention and scientific research are very helpful as they contribute to an understanding about cause-effect relationships between gait stability and coordination and STF. Most of the training programs available, however, are closely related to the investigations at hand and cannot be applied to training situations in the operational practice for the prevention of STF accidents. An emphasis seems to be rather given to prevention areas related to slipping and falling while obstacle avoidance or tripping prevention could be assumed to be an unknown quantity, if there were not some research groups addressing relevant issues. However, more research is required to provide evidence for effective interventions.

Preliminary information about ongoing investigations allowed to inform about initial results. It could be demonstrated that the study on learning effects of gait stability in young adults in a virtual obstacle avoidance training was well received by the participants. Although, results on biomechanical data is not yet available, the positive assessments of VR techniques in action in the training program provides a sound basis for guiding similar and subsequent studies along lessons learned and experiences gained in the present study and training programs available.

\section{References}

1. DGUV: Statistik. Arbeitsunfallgeschehen 2018. Deutsche Gesetzliche Unfallversicherung (DGUV), Berlin (2019). [publikationen.dguv.de/widgets/pdf/download/article/3680]

2. Lehto, M.R., Cook, B.T.: Occupational health and safety management. In: Salvendy, G. (ed.) Handbook of human factors and ergonomics, pp. 701-733. Wiley, Hoboken (2012)

3. EU OSH Framework Directive 89/391/EEC of 12 June 1989 on the introduction of measures to encourage improvements in the safety and health of workers at work (with amendments 2008). Official Journal of the European Union L 183, 29/06/1989, 1-8 (2008)

4. Simeonov, P.: Fall risk associated with restricted and elevated support surfaces. In: Hsiao, H. (ed.) Fall prevention and protection. Principles, guidelines, and practices, pp. 119-140. CRC Press, Boca Raton (2017)

5. Simpson, B.D., Cowgill, J.L., Gilkey, R.H., Weisenberger, J.M.: Technological considerations in the design of multisensory virtual environments: How real does it need to be? In: Hale, K.S., Stanney, K.M. (eds.) Handbook of virtual environments: Design, implementation, and applications, pp. 313-333. CRC Press, Boca Raton (2015)

6. Champney, R.K., Carroll, M., Surpris, G., Cohn, J.: Conducting training transfer studies in virtual environments. In: Hale K.S., Stanney K.M. (eds.) Handbook of virtual environments: Design, implementation, and applications, pp. 781-795. CRC Press, Boca Raton (2015)

7. Gordon, D., Robertson, E. Caldwell, G.E. Hamill, J. Kamen, G., Whittlesey, S.N. (eds.): Research methods in biomechanics. Human Kinetics, Champaign (2014).

8. Sheik-Nainar, M.A., Kaber, D.B.: The utility of a virtual reality locomotion interface for studying gait behavior. Human Factors 49(4), 696-709 (2007).

9. Arnold, R.: Assisted learning. A workbook. Bildungstransfer Verlag, Landau (2010).

10. Safety in a Box, www.worksafetexas.com/videos/safety-in-a-box.aspx, last accessed 2020/01/30 
11. Plonsker, T.: Lernen in und mit virtuellen Welten. DGUV Forum 4, 36-37 (2019).

12. DGUV Forum, https://www.dguv-forum.de/files/594/19-50035_DGUV_Forum_4_2019_screen.pdf, last accessed 2020/01/30

13. Safety and health at work, www.healthandsafetyatwork.com/feature/working-height-safetylondon-office-block, last accessed 2019/12/15

14. American Society of Safety Professionals, www.assp.org/news-and-articles/2018/07/10/using-virtual-reality-as-a-fall-protection-training-tool, last accessed 2020/01/30

15. Savannah River Site, www.energy.gov/em/articles/srs-liquid-waste-contractor-uses-virtualreality-slips-trips-and-falls-training, last accessed 2020/01/30

16. BGHW: In diesem Lkw steckt was drin. BGHW aktuell 3, 18-21 (2019).

17. BGHW, https://www.bghw.de/medien/bghw-aktuell-die-zeitschrift-fuer-mitgliedsbetriebe/bghw-aktuell-03-19/bghw-aktuell-3-19, last accessed 2020/01/30

18. VR Health and Safety Training, www.virtualrealityexps.com/vr-health-and-safety/, last accessed 2020/01/30

19. IBEW, www.bitspacedevelopment.com/ibew-slips-trips-falls/, last accessed 2020/01/15

20. EdgVR, www.edg-vr.com/, last accessed 2020/01/15

21. Giotakos, O., Tsirgogianni, K., Tarnanas, I.: A virtual reality exposure therapy (VRET) scenario for the reduction of fear of falling and balance rehabilitation training of elder adults with hip fracture history. Virtual Rehabilitation, 155-158 (2007). https://doi.org/10.1109/icvr.2007.4362157

22. Parijat, P., Lockhart, T.E., Liu, J.: Effects of perturbation-based slip training using a virtual reality environment on slip-induced falls. Annals of Biomedical Engineering 43(4), 958967. (2014) https://doi.org/10.1007/s10439-014-1128-z

23. Parijat, P., Lockhart, T.E., Liu, J.: EMG and kinematic responses to unexpected slips after slip training in virtual reality. IEEE Transactions on Biomedical Engineering, 62(2), 593599 (2015). https://doi.org/10.1109/tbme.2014.2361324

24. Riem, L., Van Dehy, J., Onushko, T., Beardsley, S.: Inducing compensatory changes in gait similar to external perturbations using an immersive head mounted display. In Proceedings of the 2018 IEEE Conference on Virtual Reality and 3D User Interfaces (VR), 128-135. (2018). https://doi.org/10.1109/vr.2018.8446432

25. Menegoni, F., Albani, G., Bigoni, M., Priano, L., Trotti, C., Galli, M., Mauro, A.: Walking in an immersive virtual reality. Studies in Health Technology and Informatics 144 (Annual Review of Cybertherapy and Telemedicine), 72-76 (2009). https://doi.org/10.3233/978-160750-017-9-72

26. Martelli, D., Xia, B., Prado, A., Agrawal, S.K.: Gait adaptations during overground walking and multidirectional oscillations of the visual field in a virtual reality headset. Gait \& Posture 67, 251-256 (2019). https://doi.org/10.1016/j.gaitpost.2018.10.029

27. Liu, J., Lockhart, T., Parijat, P., McIntosh, J.D., Chiu, Y.P.: Comparison of slip training in vr environment and on moveable platform. In 52nd Annual Rocky Mountain Bioengineering Symposium and 52nd International ISA Biomedical Sciences Instrumentation Symposium 2015, 192-200. International Society of Automation (ISA) (2015).

28. Peterson, S.M., Rios, E., Ferris, D.P.: Transient visual perturbations boost short-term balance learning in virtual reality by modulating electrocortical activity. Journal of Neurophysiology 120(4), 1998-2010 (2018). https://doi.org/10.1152/jn.00292.2018

29. Nyberg, L., Lundin-Olsson, L., Sondell, B., Backman, A., Holmlund, K., Eriksson, S., Stenvall, M., Rosendahl, E., Maxhall, M., Bucht, G.: Using a virtual reality system to study balance and walking in a virtual outdoor environment: A pilot study. CyberPsychology \& Behavior 9(4), 388-395 (2006). https://doi.org/10.1089/cpb.2006.9.388 
30. Kim, A., Schweighofer, N., Finley, J.M.: Locomotor skill acquisition in virtual reality shows sustained transfer to the real world. Journal of NeuroEngineering and Rehabilitation 16(1), 113-123 (2019). https://doi.org/10.1186/s12984-019-0584-y

31. Kennedy, R.S., Berbaum, K.S., Lilienthal, M.G.: Simulator Sickness Questionnaire: An enhanced method for quantifying simulator sickness. The International Journal of Aviation Psychology 3(3), 203-220 (1993)

32. Pfendler, C., Thun, J.: Der Simulator Sickness Questionnaire von Kennedy et al. (1993) und seine rechnergestützte Version (Technischer Bericht). Forschungsinstitut für Kommunikation, Informationsverarbeitung und Ergonomie (FKIE), Wachtberg (2001)

33. Witmer B.G., Singer, M.J.: Measuring presence in virtual environments: A presence questionnaire. Presence, 7(3), 225-240 (1998)

34. Witmer, B.G., Jerome, C.J., Singer, M.J.: The factor structure of the Presence Questionnaire. Presence 14(3), 298-312 (2005)

35. Hart, S.G., Staveland, L.E.: Development of the NASA task load index (TLX): Results of empirical and theoretical research. In: Hancock, P.A., Meshkati, N. (eds.) Human mental workload, pp. 139-183. North-Holland, Amsterdam (1988)

36. Stanney, K.M., Kennedy, R.S., Drexler, J.M.: Cybersickness is not simulator sickness, Proceedings of the 41st Annual Meeting of the Human Factors and Ergonomics Society (HFES 1997), pp. 1138-1142, Sept 22-26, 1997, Albuquerque. HFES, San Diego (1997)

37. Weber, A., Nickel, P., Hartmann, U., Friemert, D., Karamanidis, K.: Capture of stability and coordination indicators in virtual training scenarios for the prevention of slip, trip, and fall (STF) accidents. Lecture Notes in Computer Science (LNCS) 11581, 210-219 (2019). [doi.org/10.1007/978-3-030-22216-1_16]

38. Weber, A., Hartmann, U., Friemert, D., Epro, G., Seeley, J., Nickel, P., Karamanidis, K.: A virtual reality obstacle avoidance task leads to limb-specific locomotor adaptations but not interlimb transfer. Manuscript under preparation. 